JOURNAL OF COMPUTATIONAL PHYSICS 143, 495-518 (1998)

ARTICLE NO. CP975810

\title{
Capturing the Behavior of Bubbles and Drops Using the Variational Level Set Approach ${ }^{1}$
}

\author{
Hong-Kai Zhao, ${ }^{2}$ Barry Merriman, Stanley Osher, and Lihe Wang \\ Department of Mathematics, University of California, Los Angeles, Los Angeles, California 90095-1555
}

Received October 18, 1996; revised July 10, 1997

\begin{abstract}
We reproduce the general behavior of complicated bubble and droplet motions using the variational level set formulation introduced by the authors earlier. Our approach here ignores inertial effects; thus the motion is only correct as an approximation for very viscous problems. However, the steady states are true equilibrium solutions. Inertial forces will be added in future work. The problems include: soap bubbles colliding and merging, drops falling or remaining attached to a (generally irregular) ceiling, and liquid penetrating through a funnel in both two and three dimensions. Each phase is identified with a particular "level set" function. The zero level set of this function is that particular phase boundary. The level set functions all evolve in time through a constrained gradient descent procedure so as to minimize an energy functional. The functions are coupled through physical constraints and through the requirements that different phases do not overlap and vacuum regions do not develop. Both boundary conditions and inequality constraints are cast in terms of (either local or global) equality constraints. The gradient projection method leads to a system of perturbed (by curvature, if surface tension is involved) HamiltonJacobi equations coupled through a constraint. The coupling is enforced using the Lagrange multiplier associated with this constraint. The numerical implementation requires much of the modern level set technology; in particular, we achieve a significant speed up by using the fast localization algorithm of H.-K. Zhao, M. Kang, B. Merriman, D. Peng, and S. Osher. (c) 1998 Academic Press
\end{abstract}

\section{INTRODUCTION}

In this article we shall develop a class of algorithms to capture the behavior of multiphase bubbles and drops in two and three space dimensions. We include some very interesting and recently analyzed steady state cases—e.g. [8], where "double bubbles minimize." This general class of problems has recently received a lot of attention [19].

${ }^{1}$ Research supported by ARPA/ONR N00014-92-J-1890, NSF DMS94-04942, and ARO DAAH04-95-1-0155

${ }^{2}$ H. K. Zhao is currently at: Mathematics Department, Stanford University, Stanford, CA 94305-2125. 
We shall use the level set method, first developed in [15], which has been successfully applied to a variety of problems, in order to capture the evolution of complex interfaces in fluid dynamics and elsewhere (see, e.g., $[13,14]$ and the references therein). Topological changes and the development of singularities pose no difficulties for this method.

As initially designed in [15], the level-set method applied naturally to problems in which there is a clear distinction between "inside" and "outside" of a (possibly multiply connected) region. Two phase flow problems coupling the motion to the full Navier-Stokes of Euler equations [2,21] or to heat release [3], as well as unstable vortex sheet motion [5] and other unstable fronts [6], were recently solved using this method, extending its utility beyond the geometry-driven motion of the original paper [15]. For two-phase immiscible problems, the zero level set of a single function evolves as the interface (perhaps inducing topological changes).

In the general (at least three phase) multiphase case a new methodology is needed. In [12], Merriman, Bence, and Osher first extended the level set method to compute the motion of multiphase junctions. Also in that paper, and in $[10,11]$ a simple method based on the diffusion of characteristic functions followed by a simple reassignment step, was shown to be appropriate for the motion of multiple junctions corresponding to pure mean curvature flow. More general motion involving rather arbitrary functions of curvature, perhaps different for each interface was developed in [12] as well. While the method in [12] was not restricted to gradient flows, it lacks (so far) a clear theoretical basis.

In [18] another approach was suggested in which an influence matrix between each pair of phases has to be built à priori. In real problems this matrix can be very complicated and may not be determined beforehand. The normal velocity may depend on local quantities such as curvature, normal direction, as well as global quantities and constraints such as incompressibility, vortex sheet strength, etc. Moreover, the method in [18] requires $n(n-1)$ level set functions.

Our method is based on the variational level set approach developed in [22]. As in [12], we need $n$ level set functions - as many as there are phases. We associate the system with a physically meaningful energy functional. A gradient flow is defined; this determines the normal velocity at the interface. The $n$ level set functions are coupled through local and/or global constraints (usually both). This formulation gives us the ability to associate each phase with its different physical properties, e.g., surface tension, density, bulk energy, etc. Also, boundary conditions and inequality constraints can be turned into equality constraints which we incorporate easily into the algorithm.

We use this formulation here in order to model several interesting multiphase phenomena in both two and three dimensions. These include: several soap bubbles colliding and merging, drops falling from a ceiling and pinching off, drops sitting on a table, and fluid flowing through a narrow funnel. Our numerical results validate certain expected differences between two and three dimensions [19].

We note that the motion is that induced by using gradient descent on the potential energy; inertial forces are not included. Nevertheless, steady states computed this way involving complicated multiphase configurations are correct, as is the motion for unsteady viscous dominated flows. Inertial forces will be included through a level set based Hamilton's principle formulation in our future work; see also [9].

We also note that Chopp [4], in related work, has constructed minimal surfaces in $R^{3}$ attached to given curves by evolving via level sets and mean curvature flow. He enforces the 
boundary conditions by repeatedly reattaching the surface to the boundary. This method is different from our present approach which uses constrained optimization.

\section{PRELIMINARIES: REVIEW OF THE VARIATIONAL LEVEL SET FORMULATION}

In this approach we express the potential energy of the system having $n$ phases in terms of the $n$ level set functions.

Let $\varphi_{i}(\bar{x})$ be the level set associated with the phase which occupies region $\Omega_{i}$. Here $\bar{x}=\left(x_{1}, x_{2}\right)$ or $\bar{x}=\left(x_{1}, x_{2}, x_{3}\right), \Omega_{i}$ might be multiply connected, and $\partial \Omega_{i}=\left\{\bar{x} / \varphi_{i}(\bar{x})=0\right\}$.

Examples of quantities which make up the energy associated with this phase are: the bulk energy,

$$
\mathcal{E}_{B}^{(i)}=\int \rho(\bar{x}) H\left(\varphi_{i}(\bar{x})\right) d \bar{x}
$$

where $\rho \geq 0$ is a density function and $H(x)$ for $x \varepsilon R^{1}$ is the Heaviside function; the gravitational potential energy,

$$
\mathcal{E}_{G}^{(i)}=-\int \mathbf{g} \cdot \bar{x} \rho(\bar{x}) H\left(\varphi_{i}(\bar{x})\right) d \bar{x},
$$

where $\mathbf{g}$ is the magnitude and direction of the gravitational force; the surface energy,

$$
\mathcal{E}_{\mathcal{I}}^{(i)}=\int \delta\left(\varphi_{i}(\bar{x})\right)\left|\nabla \varphi_{i}(\bar{x})\right| \gamma_{i}(\bar{x}) d \bar{x}
$$

where $\delta(x)=(d / d x) H(x)$ is the Dirac delta function, and $\gamma_{i}$ the interfacial surface tension.

As mentioned above, the phases evolve so as to satisfy certain constraints. The central constraint for level set based multiphase motion is that the phases do not overlap, and vacuum regions do not develop. This can be expressed as

$$
\sum_{i=1}^{n} H\left(\varphi_{i}(\bar{x}, t)\right)-1=0 \quad \forall \bar{x}
$$

This is an uncountably infinite set of pointwise constraints and is, thus, impractical to enforce. Instead we try to replace (2.2) by a single constraint:

$$
\left.\frac{1}{2} \iint\left(\sum_{i=1}^{n} H\left(\varphi_{i}(\bar{x}, t)\right)-1\right)\right)^{2} d \bar{x}=0 .
$$

This was shown in [22] to result in a degenerate constraint; i.e. the gradient of the constraint functional vanishes on the constraint set. This makes it unsuitable for use with Lagrange multipliers. Instead, we require that

$$
\frac{1}{2} \iint\left(\sum_{i=1}^{n} H\left(\varphi_{i}(\bar{x}, t)\right)-1\right)^{2} d \bar{x}=\epsilon
$$


for $\epsilon>0$ as small as we can manage numerically. In the triple point motion of [22] we found $\epsilon$ corresponded to the area (or volume) of one grid cell. In this paper $\epsilon$ corresponds to, at most, the area (or volume) of a few grid cells.

In the case of incompressible fluids, the area (volume) of each bubble or drop is conserved. This amounts to requiring

$$
\int_{\Omega} \rho(\bar{x}) H\left(\varphi_{i}(\bar{x})\right) d \bar{x} \equiv C .
$$

(Throughout this paper, $\epsilon$ will denote any small positive constant and $C$ will denote any $O$ (1) positive constant.)

The variational level set formulation of any of our multiphase problems, is thus of the form:

Minimize

$$
\mathcal{E}=\int f\left(\varphi_{1}(\bar{x}), \varphi_{2}(\bar{x}), \ldots, \varphi_{n}(\bar{x})\right) d \bar{x}
$$

subject to the constraints

$$
\int g_{i}\left(\varphi_{1}(\bar{x}), \varphi_{2}(\bar{x}), \ldots, \varphi_{n}(\bar{x})\right) d \bar{x}=C_{i}, \quad i=1, \ldots, m .
$$

Using the gradient projection method of Rosen [17], we obtain the coupled system of evolution equations,

$$
\frac{\partial \varphi_{i}}{\partial t}=-\frac{\partial f}{\partial \varphi_{i}}-\sum_{j=1}^{n} \lambda_{j} \frac{\partial g_{j}}{\partial \varphi_{i}}, \quad i=1, \ldots, n,
$$

where each $\lambda_{j}$ is a Lagrange multiplier.

The constraints satisfy

$$
\frac{d}{d t} \int_{\Omega} g_{j}\left(\varphi_{1}, \varphi_{2}, \ldots, \varphi_{n}\right) d \bar{x}=0, \quad j=1, \ldots, m,
$$

which determines the Lagrange multipliers as solutions of the linear system

$$
\begin{gathered}
\sum_{k=1}^{m} \lambda_{k} \int_{\Omega}\left(\nabla_{\varphi} g_{j} \cdot \nabla_{\varphi} g_{k}\right) d \bar{x}=-\int\left(\nabla_{\varphi} g_{j} \cdot \nabla_{\varphi} f\right) d \bar{x} \\
j=1, \ldots, m, \text { for } \phi=\left(\varphi_{1}, \ldots, \varphi_{m}\right) .
\end{gathered}
$$

It is easy to see that (a) this system of equations defined in (2.11) is nonsingular if $\left(\nabla_{\varphi} g_{1}, \ldots, \nabla_{\varphi} g_{m}\right)$ are linearly independent, and (b) the energy functional is nonincreasing in that case and, furthermore, is strictly decreasing if $\left(\nabla_{\varphi} g_{1}, \ldots, \nabla_{\varphi} g_{m}, \nabla_{\varphi} f\right)$ are also linearly independent.

This system of evolution equations often contains Hamilton-Jacobi equations coupled to curvature and stiff source terms. Singularities may develop in the solution. The numerical implementation requires much of the modern level set technology. See [22] and Section 4 below for details. The crucial ingredients are: 
(i) High order accurate essentially nonoscillatory schemes (originating in the study of hyperbolic conservation laws [7, 20]) developed for Hamilton-Jacobi equations in [15, 16].

(ii) Reinitialization of each of the level set functions to be the signed distance to the appropriate interface. This can be easily done by interspersing a few iterations of the following nonlinear partial differential evolution equation,

$$
\left(d_{i}\right)_{\tau}+\operatorname{sign}\left(\varphi_{i}\right)\left(\left|\nabla d_{i}\right|-1\right)=0, \quad i=1, \ldots, m,
$$

with the evolution procedure and then replacing $\varphi_{i}$ by $d_{i}$. This idea originated in [21].

(iii) Using the distance function to define curvature on or near the front in the defining Eqs. (2.11) for the Lagrange multipliers via

$$
\kappa_{i}=\operatorname{trace}\left[\left[I-d D^{2} d\right]^{-1} D^{2} d\right],
$$

where $D^{2} d$ is the Hessian of $d_{i}$.

This formula yields a constant value of $\kappa_{i}$ normal to the front and, of course, is correct on the front.

To speed up the level set methods, particularly in three dimensions, we have developed a robust localization technique which only requires computation in a very narrow tube (at most two grid points wide) near the front [23]. An earlier approach was developed in [1]. Ours works easily for multiphase problems in two and three dimensions and in the presence of topological changes. The computational cost is linearly proportional to the number of points on the front, which is optimal. This method essentially consists of moving the tube with the motion of the front and reinitializing the level set function only in the tube. (See Fig. 1). Everything is done in terms of the values of $d_{i}$ for $\left|d_{i}\right|<\epsilon$, where $\epsilon$ is $\leq 2 \Delta x$. No "exploring" in $\bar{x}$ space is required. We use an upwind scheme in the reinitialization step, thus avoiding any need for numerical boundary conditions at the boundary of the tube.

To summarize: The variational level set formulation using the gradient projection method is applicable to a wide variety of complicated problems of both physical and mathematical interest. Its virtues are:

(a) The method is quite stable since the associated energy diminishes in time.

(b) The level set method deals with topological changes, kinks, cusps, and the computation of geometric properties of the front, such as curvature and unit normal, very naturally in both two and three dimensions.

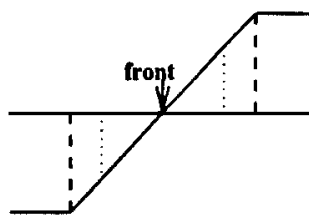

(o) Inittal signed distance

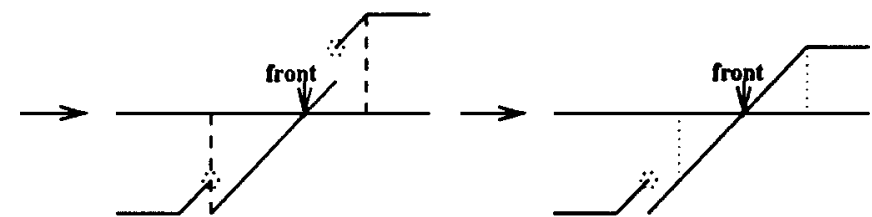

(b) motion of the front and tube (c) after reinitinlization in the tube boundary of the tube in which level set is updated

boundary of the tube in which level set is reinitialized

level set function

FIG. 1. Local level set method. 
(c) Constraints (including boundary conditions) are easily incorporated.

(d) Recently developed accurate and nonoscillatory numerical methods are now available for solving the resulting equations of motion.

(e) The fast localization technique [23] speeds up the calculations and makes threedimensional problems accessible on workstations.

\section{VARIATIONAL FORMULATIONS FOR MULTIPHASE FLUID PROBLEMS}

\subsection{Soap Bubbles}

Suppose we have $(n-1)$ nonoverlapping bubbles $B_{1}, B_{2}, \ldots, B_{n-1}$ in the region $\Omega$. Each $B_{i}$ is associated with a function $\varphi_{i}(\bar{x})$ with $\varphi_{i}(\bar{x})>0$ if $\bar{x} \varepsilon B_{i}, \varphi_{i}(\bar{x})<0$ if $\bar{x} \phi\left(B_{i} \cup\right.$ $\left.\partial B_{i}\right), \varphi_{i}(\bar{x})=0$ if $\bar{x} \varepsilon \partial B_{i}$. We define $\phi_{0}(\bar{x})$ to be the level set function associated with the region exterior to all bubbles:

$$
\Omega-\bigcup_{i=1}^{n-1}\left(B_{i} \cup \partial B_{i}\right)
$$

i.e., $\varphi_{0}(\bar{x})>0$ for $\bar{x}$ in the interior of that region, $\varphi_{0}(\bar{x})<0$ for $\bar{x}$ in any one of the bubbles, $\varphi_{0}(\bar{x})=0$ for $\bar{x}$ on the boundary of any one of the bubbles.

We first ignore gravity and consider only the energy due to surface tension. We assume that each bubble conserves its area (volume). Together with our general multiphase requirement (2.3) or (2.4), we have the following variational level set formulation (which is identical with that used in our work on optimal domain decomposition [24]):

Minimize the surface energy

$$
\mathcal{E}=\sum_{i=0}^{n-1} \mathcal{E}_{\mathcal{I}}^{(i)}=\sum_{i=0}^{n-1} \int \gamma_{i} \delta\left(\varphi_{i}(\bar{x})\right)\left|\nabla \varphi_{i}(\bar{x})\right| d \bar{x}
$$

subject to nonoverlap and conservation of volume constraints,

$$
\begin{gathered}
\frac{1}{2} \int_{\Omega}\left(\sum_{i=0}^{n-1} H\left(\varphi_{i}(\bar{x})\right)-1\right)^{2} d \bar{x}=\epsilon \\
\int H\left(\varphi_{i}(\bar{x})\right) d x=A_{i}, \quad i=1,2, \ldots, n-1 .
\end{gathered}
$$

In this formulation, at the interface $\Gamma_{i j}$ between phase $i$ and $j$, the total surface tension is $\gamma_{i}+\gamma_{j}$. Different sets of $\left\{\gamma_{i}\right\}_{i=0}^{n-1}$ give different physical problems. If, for example, we take all $\gamma_{i}=0$ for $i=1, \ldots, n-1$ and $\gamma_{0}=1$, then all the bubbles which touch initially will merge into one big circle (sphere) and the steady state will be a family of circles or spheres. This is a problem for which the surface tension between any two bubbles is zero and that between any bubble and the air is 1 .

Another approach to this special, interesting problem was taken in [9] using only one level set, thus requiring some decisions at merging. However, that paper also included inertial forces so that the dynamics was time accurate, causing bubbles to vibrate, as they should. We shall handle this situation in the future through a level set version of Hamilton's variational principle. 
Another interesting case occurs when $\gamma_{0}=\gamma_{1}=\gamma_{2}=0.5$ for two bubbles which initially touch. Then the interface between the two bubbles and the interface between each bubble and the air has surface tension one. In the three-dimensional version of two bubbles with the same volume, a long standing conjecture was proven in [8], i.e. that two spheres with a disc as the common interface are the global minimizer. This solution is realized in our calculations, as seen in Figs. 7 and 9.

We use the projection-gradient method, rescale (as in [22]), and arrive at the time evolution equations

$\frac{\partial \varphi_{i}}{\partial t}(\bar{x}, t)=\left|\nabla \varphi_{i}(\bar{x}, t)\right|\left[\nabla \cdot \gamma_{i}\left(\frac{\nabla \varphi_{i}(\bar{x}, t)}{\left|\nabla \varphi_{i}(\bar{x}, t)\right|}\right)-\mu_{i}-\lambda\left(\sum_{j=0}^{n-1} H\left(\varphi_{j}(\bar{x}, t)\right)-1\right)\right]$,

$i=0,1, \ldots, n-1, \mu_{0}=0$, with boundary conditions

$$
\frac{\partial \varphi_{i}}{\partial n}(\bar{x})=0 \quad \text { on } \Omega .
$$

The Lagrange multipliers are $\mu_{i}, i=1, \ldots, n-1$, corresponding to ( $\left.3.2 \mathrm{~b}\right)$, and $\lambda$ corresponding to (3.2a). They are determined from (2.11), properly scaled, by the linear system

$$
\left[m_{i j}\right]_{n \times n} \cdot\left[\begin{array}{c}
\lambda \\
\mu_{1} \\
\vdots \\
\mu_{n-1}
\end{array}\right]=\left[\begin{array}{c}
b_{1} \\
b_{2} \\
\vdots \\
b_{n}
\end{array}\right],
$$

where

$$
\begin{aligned}
m_{11}= & \sum_{i=0}^{n-1} \int_{\Omega} \delta\left(\phi_{i}(\bar{x}, t)\right)\left|\nabla \phi_{i}((\bar{x}, t))\right|\left(\sum_{j=0}^{n-1} H\left(\phi_{j}((x, t))\right)-1\right)^{2} d \bar{x} \\
m_{i i}= & \int_{\Omega} \delta\left(\phi_{i-1}(\bar{x}, t)\right)\left|\nabla \phi_{i-1}(\bar{x}, t)\right| d \bar{x}, \quad i=2, \ldots, n, \\
m_{i 1}= & m_{1 i}=\int_{\Omega} \delta\left(\phi_{i-1}(\bar{x}, t)\right)\left|\nabla \phi_{i-1}(\bar{x}, t)\right|\left(\sum_{j=0}^{n-1} H\left(\phi_{j}(\bar{x}, t)\right)-1\right) d \bar{x}, \\
m_{i j}= & 0, \quad \text { otherwise; } \\
b_{1}= & \sum_{i=0}^{n-1} \int_{\Omega} \delta\left(\phi_{i}(\bar{x}, t)\right)\left|\nabla \phi_{i}(\bar{x}, t)\right|\left(\sum_{j=0}^{n-1} H\left(\phi_{j}(\bar{x}, t)\right)-1\right) \\
& \times\left[\nabla \cdot \gamma_{i}\left(\frac{\nabla \phi_{i}(\bar{x}, t)}{\left|\nabla \phi_{i}(\bar{x}, t)\right|}\right)\right] d \bar{x} \\
b_{i}= & \int_{\Omega} \delta\left(\phi_{i-1}(\bar{x}, t)\right)\left|\nabla \phi_{i-1}((\bar{x}, t))\right|\left[\nabla \cdot \gamma_{i-1}\left(\frac{\nabla \phi_{i-1}(\bar{x}, t)}{\left|\nabla \phi_{i-1}(\bar{x}, t)\right|}\right)\right] d \bar{x}, \\
i= & 2, \ldots, n .
\end{aligned}
$$


The symmetric matrix $\left[m_{i j}\right]$ is positive definite if (2.4) is satisfied (not (2.3) - that gives a degeneracy). We see that the Lagrange multiplier corresponding to the minimal overlap and/or vacuum constraint (3.2a) acts to couple the $\varphi_{i}$ so as to satisfy this crucial property.

For $\mu_{i} \neq 0$ and $\gamma_{i}>0$ and constant, then, except for the coupling constraints, we have a Hamilton-Jacobi equation corresponding to motion by a constant speed $\mu_{i}$, perturbed by $\gamma_{i}$ times the curvature. This familiar problem arises throughout the level surface world and numerical techniques and analytical results do exist [15].

We note that in the special one-bubble case a single level set is adequate-see [9]. In the present framework, if we consider (3.1)-(3.3) with any two nonnegative constants $\gamma_{0}, \gamma_{1}$, so that $\gamma_{0}+\gamma_{1}=1$, we have the same motion as in the single level set case up to a trivial time scaling. The proof of this fact is illuminating. We present it here:

$$
\begin{aligned}
& \frac{\partial \phi_{0}(\bar{x}, t)}{\partial t}=\left|\nabla \phi_{0}(\bar{x}, t)\right|\left[\gamma_{0} \nabla \cdot\left(\frac{\nabla \phi_{0}(\bar{x}, t)}{\left|\nabla \phi_{0}(\bar{x}, t)\right|}\right)-\lambda\left(\sum_{j=0}^{1} H\left(\phi_{j}(\bar{x}, t)\right)-1\right)\right] \\
& \frac{\partial \phi_{1}(\bar{x}, t)}{\partial t}=\left|\nabla \phi_{1}(\bar{x}, t)\right|\left[\gamma_{1} \nabla \cdot\left(\frac{\nabla \phi_{1}(\bar{x}, t)}{\left|\nabla \phi_{1}(\bar{x}, t)\right|}\right)-\mu_{1}-\lambda\left(\sum_{j=0}^{1} H\left(\phi_{j}(\bar{x}, t)\right)-1\right)\right] .
\end{aligned}
$$

We assume that $\sum_{i=0}^{1} H\left(\phi_{i}(\bar{x}, t)\right)-1=b \neq 0$ on the interface and denote for $i=0,1$,

$$
\int_{\Omega} \delta\left(\phi_{i}\right)\left|\nabla \phi_{i}\right| d \bar{x}=A=\text { area(length) of the interface; }
$$

$\int_{\Omega} \delta\left(\phi_{i}\right)\left|\nabla \phi_{i}\right|\left[\nabla \cdot\left(\nabla \phi_{i} /\left|\nabla \phi_{i}\right|\right)\right] d \bar{x}=K=$ total mean curvature of the interface, then (3.3(c)-(i)) here becomes

$$
\begin{aligned}
\left(2 A b^{2}\right) \lambda+A b \mu_{1} & =\left(\gamma_{1}-\gamma_{0}\right) b K \\
(A b) \lambda+A \mu_{1} & =\gamma_{1} K
\end{aligned}
$$

from which we find $\lambda=-\gamma_{0} / A b$ and, more importantly, $\mu_{1}=K / A=\bar{\kappa}=$ average mean curvature.

If we have initialized so that the $\varphi$ 's are replaced by distance functions, then $\varphi_{0}=-\varphi_{1}$ in this case and we get for each $\varphi_{i}, i=0,1$,

$$
\frac{\partial \varphi_{i}}{\partial t}(\bar{x}, t)=\frac{1}{2}\left|\nabla \varphi_{i}(\bar{x}, t)\right|\left[\nabla \cdot\left(\frac{\nabla \varphi_{i}(\bar{x}, t)}{\left|\nabla \varphi_{i}(\bar{x}, t)\right|}\right)-\bar{\kappa}\right] .
$$

This is the single level set formulation with one-half the speed.

\subsection{Drops Falling and Pinching Off from the Ceiling}

We consider a water drop initially in contact with the ceiling. The surface tension force tends to keep it attached while gravity pulls it down. A steady state shape attached to the ceiling may be obtained, or the water drop may fall. The geometric shape of the steady state solutions and the topological transitions as it leaves the ceiling are quite interesting and challenging problems-see, e.g. [19] for an interesting approach to the latter. The variational level set formulation allows us to compute the steady states (if any) accurately and also gives us a reasonable motion if acceleration affects are negligible. 


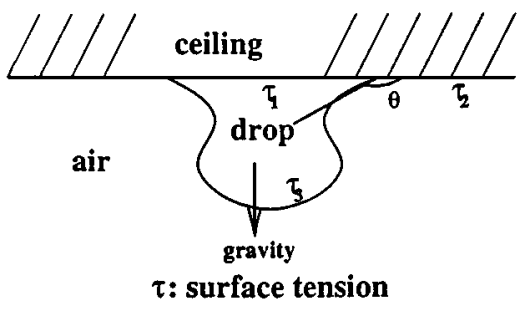

FIG. 2. Drop on the ceiling.

In Fig. 2 we show the liquid drop, the ambient air, and the ceiling, all in contact. The total energy is the surface energy plus the gravitational potential energy of the drop. The line (point) of contact of the three phases is subject to the surface tension of the three surfaces. Since it is massless, the vector resultant of the three tensions must add to zero in any direction. The contact angle satisfies $\tau_{1}-\tau_{2}=\tau_{3} \cos \theta$.

We have two constraints. The first is just that the volume (area) of the drop is preserved. The other is the boundary condition at the ceiling; i.e., the drop cannot penetrate the wall. We turn this into an equality constraint using the level set formulation. We first construct a level set function, $\psi(x)$, which is the signed distance from the ceiling. Thus the zero level set coincides with the surface of the ceiling (see Figs. 2, 3). Then we construct a surface tension function $\tau(\psi)$ defined on the zero level set of $\varphi$ :

$$
\tau(\psi)= \begin{cases}\left|\tau_{1}-\tau_{2}\right| & \text { if } \psi(x) \geq 0 \\ \tau_{3} & \text { if } \psi(x)<0\end{cases}
$$

Next we consider two cases:

Case 1. $\tau_{1}<\tau_{2}$ or $\theta>\pi / 2$. See Fig. 3(a). The liquid wets the solid with the amount of wetting increasing as $\theta$ increases to $\pi$. We initialize the configuration for the drop as in Fig. 3(a). It is easy to see that the energy functional to be minimized is composed of the surface energy of the drop,

$$
\int_{\Omega} \delta(\phi(\bar{x}))|\nabla \phi(\bar{x})| \tau(\psi) d \bar{x},
$$

and the gravitational energy of the drop,

$$
\int_{\Omega} H(\phi(\bar{x})) H(-\psi(\bar{x})) h(\bar{x}) g d \bar{x},
$$

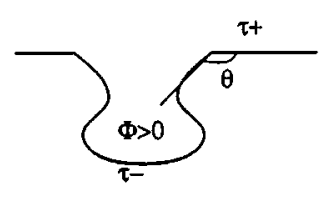

a

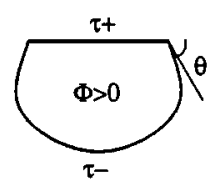

b

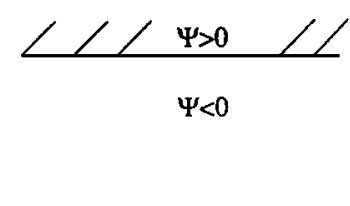

C

FIG. 3. Level sets for the drop-on-ceiling problem. 

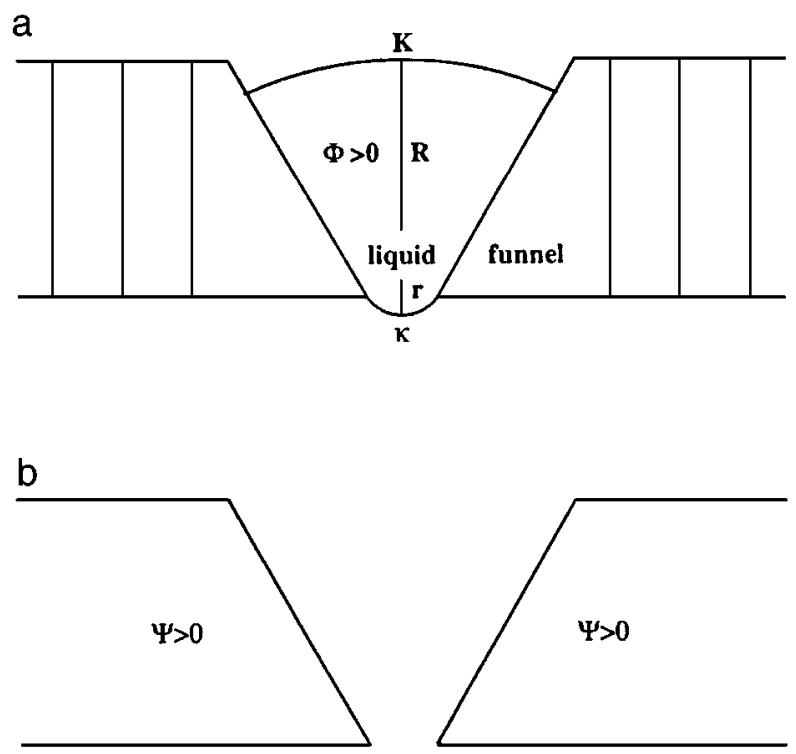

FIG. 4. (a) Liquid in a funnel; (b) funnel wall.

where $g$ is the gravity constant and $h(\bar{x})$ is the altitude. The volume (area) conservation constraint for the drop becomes

$$
\int_{\Omega} H(\phi(\bar{x})) H(-\psi(\bar{x})) d x=A
$$

and the no penetration boundary condition at the ceiling surface can be written as the constraint

$$
\int_{\Omega} H(-\phi(\bar{x})) H(\psi(\bar{x})) d \bar{x}=0 .
$$

The variational level set formulation of the problem thus becomes:

Minimize

$$
\left.\mathcal{E}=\int_{\Omega}[\delta(\phi(\bar{x})) \mid \nabla \phi(\bar{x})) \mid \tau(\psi)+H(\phi(\bar{x})) H(-\psi(\bar{x})) h(\bar{x}) g\right] d \bar{x} .
$$

Subject to

$$
\begin{aligned}
& \int_{\Omega} H(-\phi(\bar{x})) H(\psi(\bar{x})) d \bar{x}=0 \\
& \int_{\Omega} H(\phi(\bar{x})) H(-\psi(\bar{x})) d \bar{x}=A .
\end{aligned}
$$

Using the gradient projection method, we get the evolution equation for $\phi(\bar{x}, t)$

$$
\begin{aligned}
\frac{\partial \phi(\bar{x}, t)}{\partial t}= & |\nabla \phi(\bar{x}, t)|\left[\nabla \cdot\left(\tau(\psi) \frac{\nabla \phi(\bar{x}, t)}{|\nabla \phi(\bar{x}, t)|}\right)\right. \\
& -H(-\psi(\bar{x})) h(\bar{x}) g+\mu H(\psi(\bar{x}))-\lambda H(-\psi(\bar{x}))],
\end{aligned}
$$



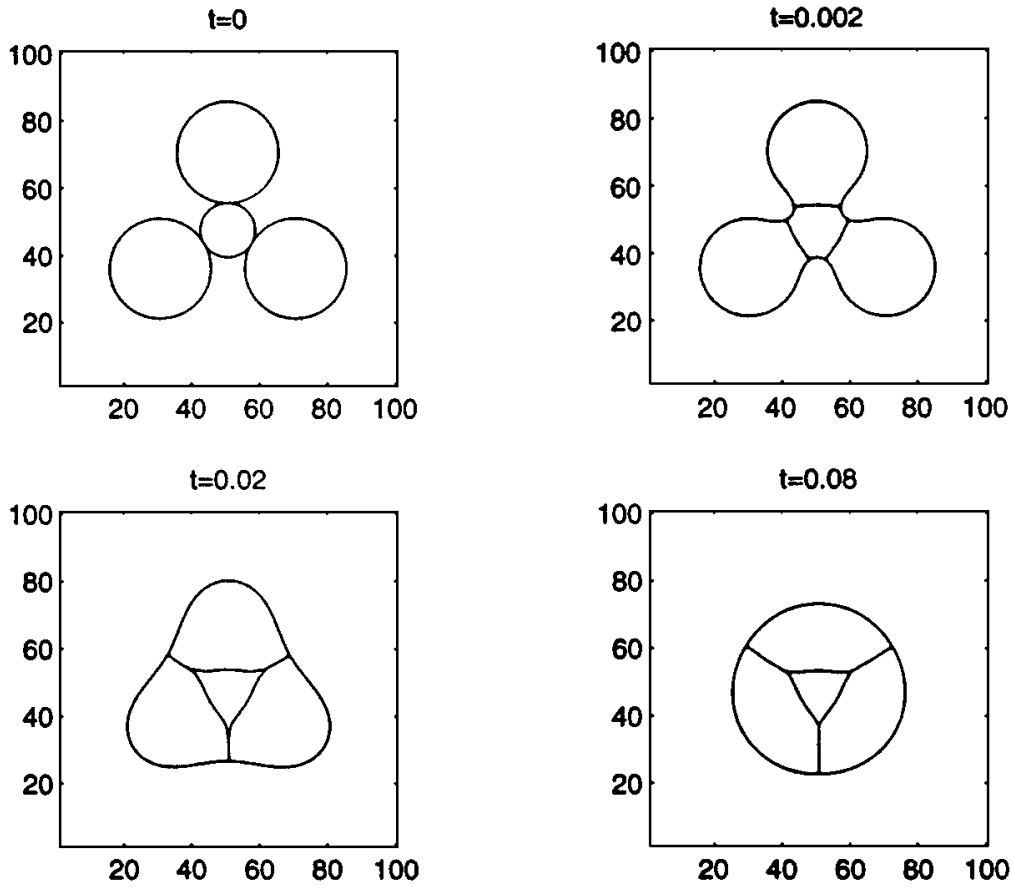

FIG. 5. Four bubbles merging in 2D. $\gamma_{0}=1, \gamma_{1}=\gamma_{2}=\gamma_{3}=\gamma_{4}=0$.
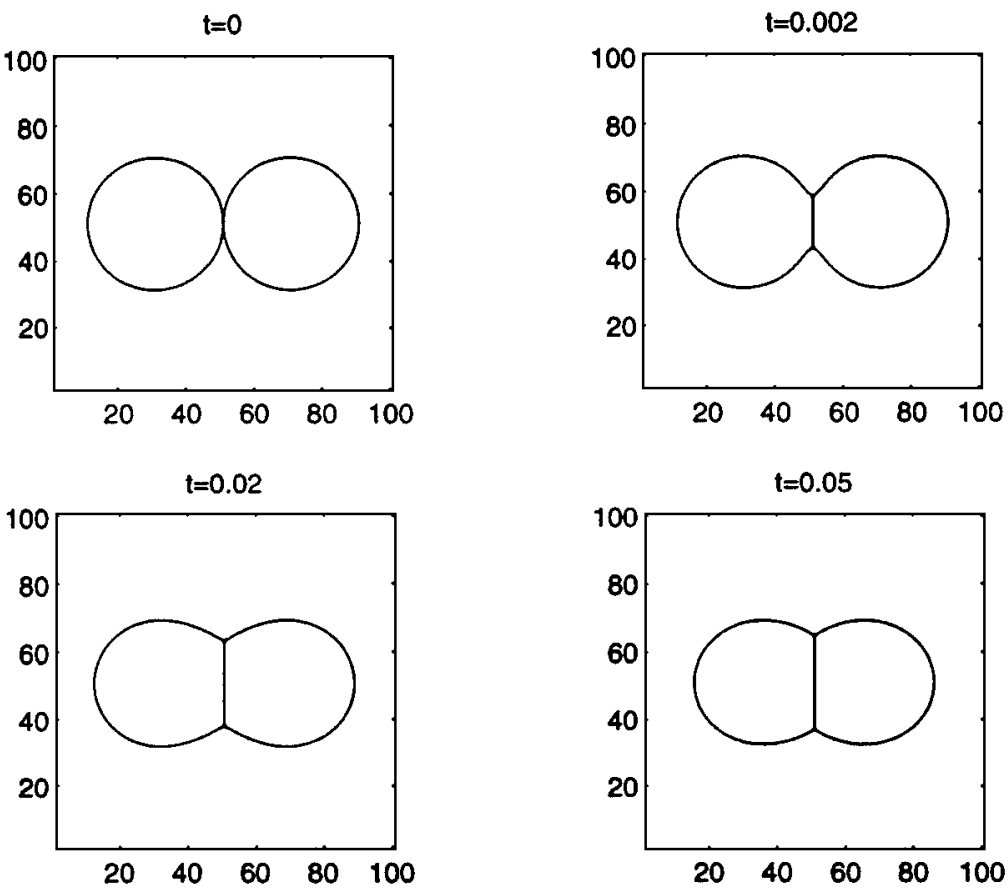

FIG. 6. Double bubble minimizer in 2D. $\gamma_{0}=\gamma_{1}=\gamma_{2}=0.5$. 

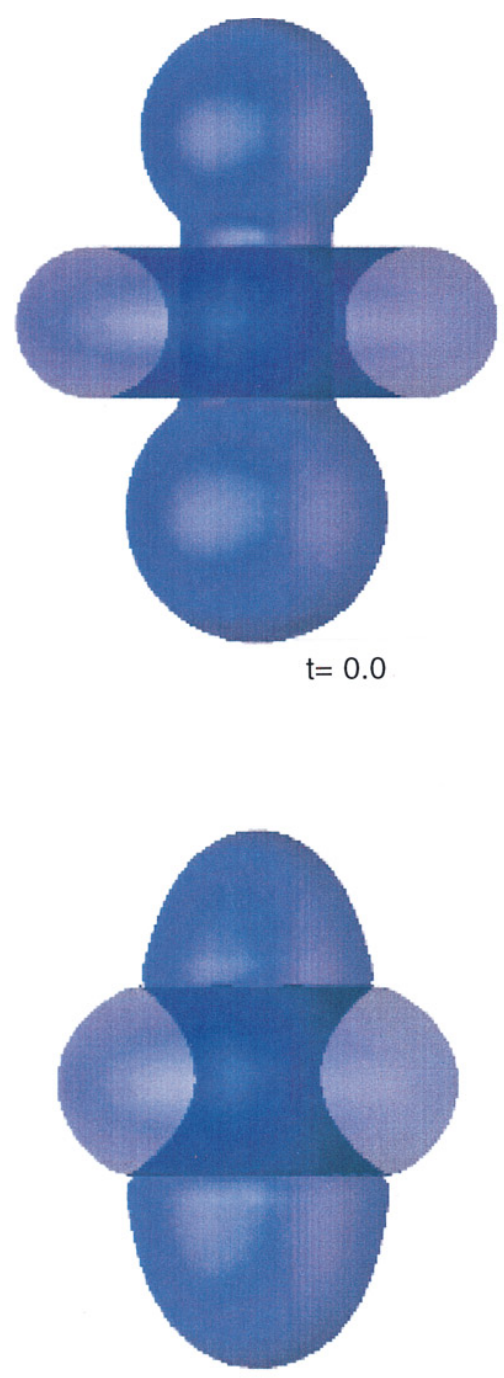

$t=0.04$

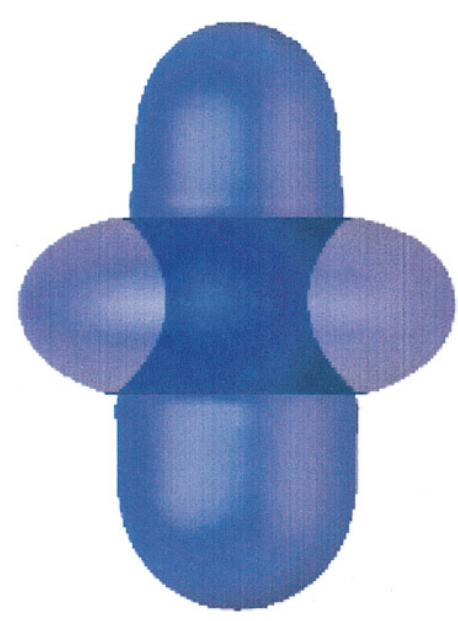

$t=0.01025$

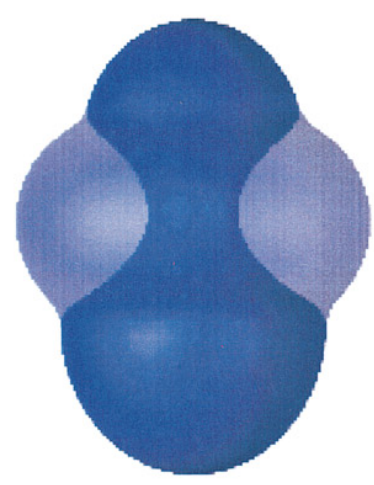

$t=0.075$

FIG. 7. Double bubble minimizer with equal surface tension.

where $\mu, \lambda$ are the Lagrange multipliers for the boundary constraint and volume (area) constraint, respectively. Using the fact that

$$
\begin{aligned}
H(\psi(\bar{x})) H(-\psi(\bar{x})) & =0, \\
H^{2}(\psi(\bar{x})) & =H(\psi(\bar{x})),
\end{aligned}
$$

we get the following decoupled linear system for the Lagrange multipliers $\lambda, \mu$ :

$$
\begin{aligned}
\mu \int_{\Omega} \delta(\phi)|\nabla \phi| H(\psi(\bar{x})) d \bar{x} & =-\int_{\Omega} \delta(\phi)|\nabla \phi| \nabla \cdot\left(\tau(\psi) \frac{\nabla \phi}{|\nabla \phi|}\right) H(\psi(\bar{x})) d \bar{x} \quad(3.10 \mathrm{c}) \\
\lambda \int_{\Omega} \delta(\phi)|\nabla \phi| H(-\psi(\bar{x})) d \bar{x} & =\int_{\Omega} \delta(\phi)|\nabla \phi|\left[\nabla \cdot\left(\tau(\psi) \frac{\nabla \phi}{|\nabla \phi|}\right)-h(\bar{x}) g\right] H(-\psi(\bar{x})) d \bar{x} .
\end{aligned}
$$




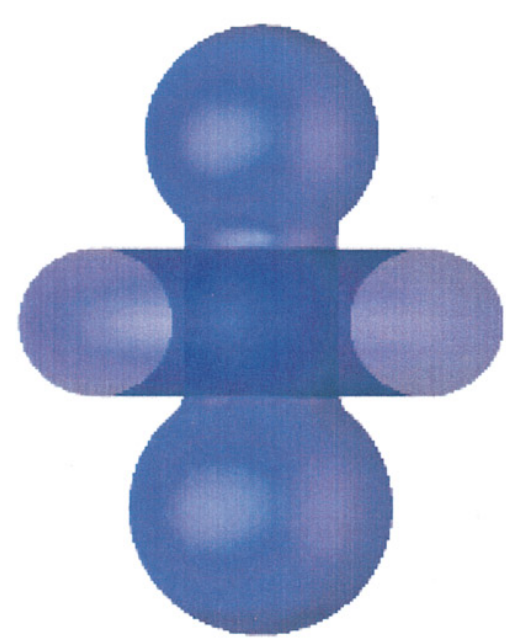

$t=0.0$

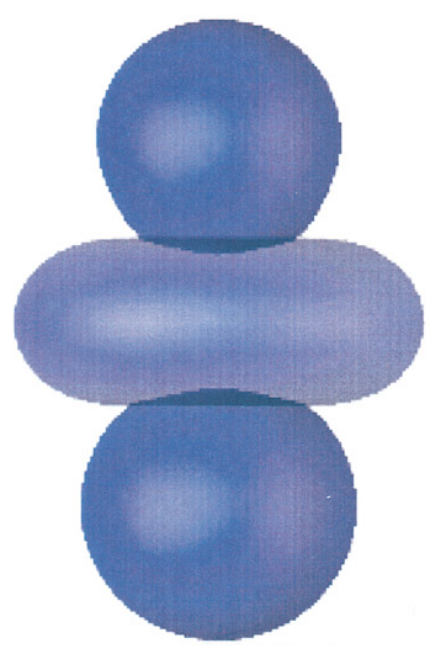

$\mathrm{t}=0.02$

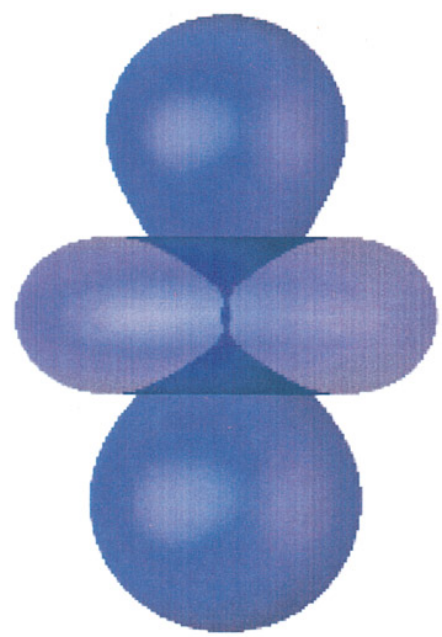

$t=0.01$

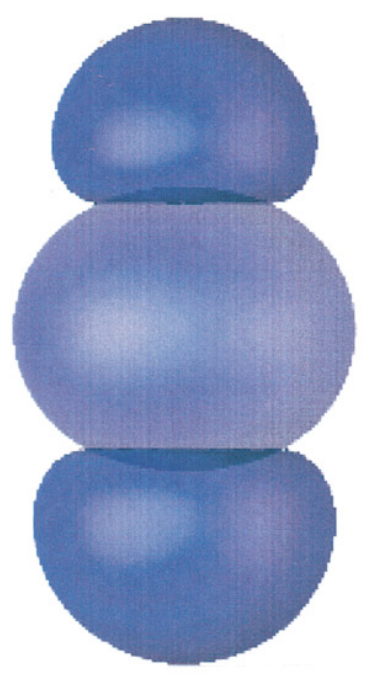

$\mathrm{t}=0.04$

FIG. 8. Double bubble minimizer with unequal surface tension.

We have obtained numerical results for the steady state shape when gravity is small or the difference of the surface tensions is large (not shown here). In the opposite case when gravity is large or the difference of the surface tensions is small, the drop falls. In three dimensions, pinchoff can occur due to gravity and the curvature effect—see Fig. 11-which is what happens in real life. In two dimensions, pinchoff occurs due solely to gravity-Figs. 10, 12, and 16. This requires a larger ratio between gravity and the difference of surface tension than in three dimensions.

Remark 3.2. We note that (3.9) and ((3.10)(d)) indicate that at steady state the shape of the drop satisfies the well-known Laplace-Young equation,

$$
\tau \nabla \cdot\left(\frac{\nabla \phi}{|\nabla \varphi|}\right)=h(\bar{x}) g
$$


i.e.,

$($ surface tension $)($ curvature $)=($ altitude $)($ gravitational constant $)$.

Case 2. $\tau_{2}<\tau_{1}$ or $\theta<\pi / 2$. (See Fig. 3(b).) This case can occur for certain liquids, e.g. mercury. Here we use a different level set configuration for the drop; see Fig. 3(b). Now the constrained minimization problem becomes formally exactly as above. Note carefully the different regions for which $\varphi>0$ in Figs. (3a) and (3b). In this case, the drop either stays on the ceiling or falls down without pinchoff. See Figs. 12, 13, and 17.

In both cases, the boundary constraint may be degenerate which means that (3.10c) just reads $0=0$ and we may set $\mu=0$ in (3.9).

Also the geometric shape of the ceiling can be quite arbitrary, e.g. a drop falling from a wedge. The only modification needed comes in the definition of $\psi(x)$-the signed distance function. Numerical results are shown in Figs. 16 and 17.

\subsection{Drops Sitting on the Floor}

If we reverse the direction of the gravity and turn the picture in Section 3.2 upside down and do the same for Figs. 3a and b, we can precisely model the liquid drop sitting on the floor case. See Figs. 14 and 15. Of course, in the unwetted wall, the drop spreads, while in the wetted wall case, the drop rises to a stationary configuration.

\subsection{Liquid Penetrating through a Narrow Funnel}

Suppose we have a narrow funnel shaped as in Fig. 4. Certain fluids flow slowly through the funnel due to gravity or some other gradient induced force. Because of the surface tension, round surfaces (arcs) are formed both on the top and bottom. The liquid may or may not go through the fluid depending on the curvature on the top and bottom, surface tension constant and weight of fluid. This model problem can be formulated almost exactly as in Section 3.2 except a more complicated barrier function $\psi(x)$ has to be constructed for the funnel as in Fig. 4, where $\psi(\bar{x})<0$ in the open complement of the region shown for which $\psi(\bar{x})>0$ :

Minimize

$$
E=\int_{\Omega} \delta(\phi(\bar{x}))|\nabla \phi(\bar{x})| \psi(\bar{x})+H(\phi(\bar{x})) h(\bar{x}) g d \bar{x},
$$

subject to

$$
\begin{aligned}
\int_{\Omega} H(\phi(\bar{x})) H(\psi(\bar{x})) d \bar{x} & =0 \\
\int_{\Omega} H(\phi(\bar{x})) H(-\psi(\bar{x})) d \bar{x} & =A .
\end{aligned}
$$

Some numerical calculations are shown in Figs. 18, 19, 20, and 21. 


\section{NUMERICAL IMPLEMENTATION AND RESULTS}

In all these examples, we have to use numerical approximations for the Heaviside function and Dirac function which are defined as

$$
\begin{aligned}
& H_{\alpha}(x)= \begin{cases}1, & x>\alpha, \\
0, & x<-\alpha, \\
\frac{1}{2}\left[1+\frac{x}{\alpha}+\frac{1}{\pi} \sin \left(\frac{\pi x}{\alpha}\right)\right], & |x| \leq \alpha ;\end{cases} \\
& \delta_{\alpha}(x)=\frac{d H_{\alpha}(x)}{d x}= \begin{cases}0, & |x|>\alpha, \\
\frac{1}{2 \alpha}\left[1+\cos \left(\frac{\pi x}{\alpha}\right)\right], & |x| \leq \alpha,\end{cases}
\end{aligned}
$$

where $\alpha$ is the numerical width of our $\delta(x)$ and $H(x)$, which we take to be the grid size $\alpha=\Delta x$.

The numerical methods developed in [22] Section 3 are directly applicable to the four problems described in Section 3 above, except for the implementation of the new constraints. For the soap bubble problem, the system of Eqs. (3.3c) has to be solved for the $\mu_{i}$ - this was also mentioned for the optimal decomposition of domain problem in [22, Eqs. (2.20)(2.21)]. No new difficulties are encountered.

For the falling drop problem we have a simple decoupled system (3.10c), (3.10d) for the two Lagrange multipliers, and the system can become degenerate because the left and right sides of (3.10c) will (and should) vanish if the drop falls. A similar situation arises in the liquid through funnel case.

This means that the no penetration constraint does not take effect until penetration occurs; then the constraint stops its progress. In our numerical calculations, we have found that the size of that penetration is at most one grid cell, as in the case for the no overlapping and no vacuum constraint. Since the values of surface tension are different for the interface between the liquid and air and for the interface between liquid and wall (wetted vs unwetted), we first defined our surface tension as

$$
\tau(\bar{x})=\tau(\psi)=\tau+\delta \tau H(-\psi(\bar{x})), \quad \bar{x} \in \Omega,
$$

where $\delta \tau$ is the difference between the two surface tensions. In our numerical calculation, we use the numerical approximation $H_{\alpha}(x)$ and, thus, cause a smoothed out transition in surface tension, within a boundary layer. We found some improvement in the numerical results, i.e. reduction of the thickness of penetration and a somewhat smoother interface when we used the following shift in the argument of the numerical Heaviside function,

$$
\tau(\bar{x})=\tau(\psi)=\tau+\delta \tau H_{\alpha}(-\psi(\bar{x})-\alpha), \quad \bar{x} \in \Omega .
$$

This is probably because the stiffest change in the surface tension is now shifted away from the boundary. Thus we used this approximation to $H(-\psi)$ in the calculations which follow.

In Fig. (5) four two-dimensional (2D) bubbles merge while the area of each bubble is preserved. We take $\gamma_{0}=1, \gamma_{1}=\gamma_{2}=\gamma_{3}=\gamma_{4}=0$. This is a real merging case since the interface length between any two bubbles does not affect the energy. We see that the inner bubbles do not become a circle. This shows that we have very little numerical viscosity. 


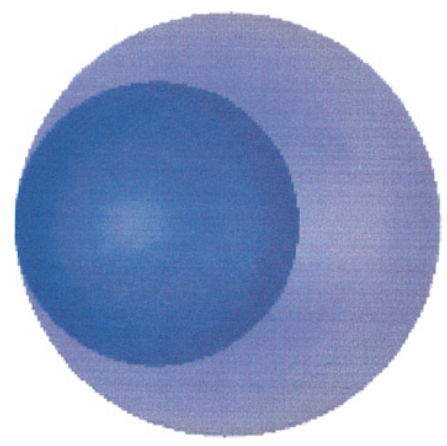

$t=0.0$

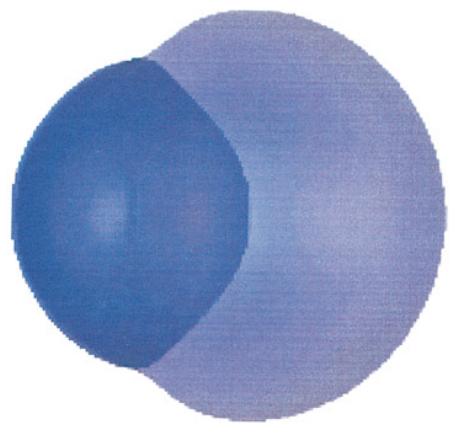

$t=0.2$

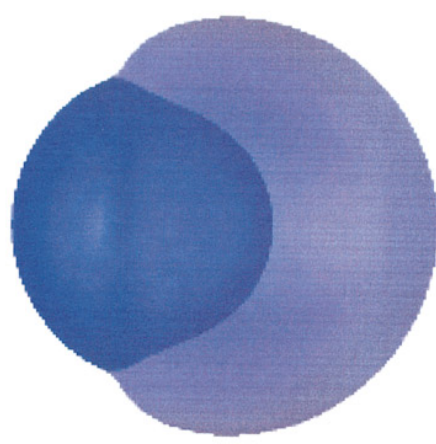

$t=0.1$

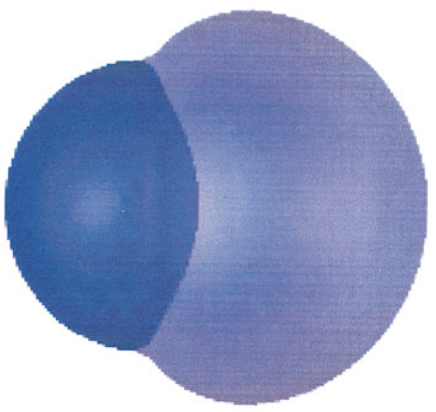

$\mathrm{t}=0.3$

FIG. 9. Double bubble minimizer with equal surface tension.

Figure 6 is a 2D double-bubble minimizer case, where $\gamma_{0}=\gamma_{1}=\gamma_{2}=0.5$; i.e., each interface has the same surface tension. We see the $120^{\circ}$ angles form at the triple point.

We next calculate bubbles merging in 3D. Figure 7 shows a dumbbell in a doughnut which is a local minimizer for the double bubble minimizer case in [8]. Here $\gamma_{0}=\gamma_{1}=\gamma_{2}=0.5$. If we let the surface tension of the dumbbell be smaller than the surface tension of the doughnut, then we see that the doughnut cuts the dumbbell in two in Fig. 8. Figure 9 is an interesting double bubble minimizer case where the smaller ball emerges from the interior of a bigger ball.

In Fig. 10, a 2D liquid (e.g., water) droplet tries to stay on the ceiling by wetting the ceiling surface as much as possible. Since gravity is large enough relative to the surface tension, we see pinchoff. Figure 11 is a similar 3D droplet calculation, but the gravity can be considerably smaller than in the 2D calculation for pinchoff to occur. Figures 12 and 13 show respectively $2 \mathrm{D}$ and $3 \mathrm{D}$ calculations corresponding to case 2 (e.g., mercury) in Section 3.2. Figures 14 and 15 are computations for the steady state shapes for drops sitting on the floor in 2D, corresponding to wetted (water) or unwetted (mercury) cases. Again the 

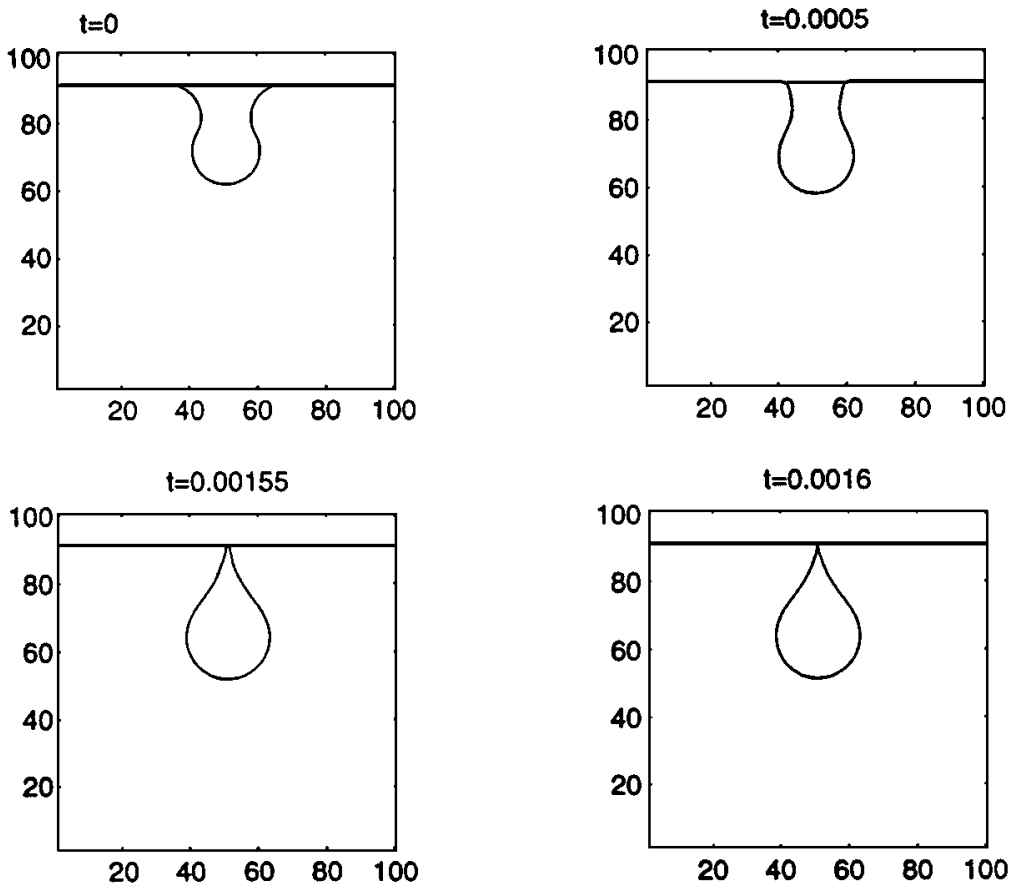

FIG. 10. Falling drop in 2 D. Surface tension with unwetted wall $=0.1$, air $=1$, and gravity $=400$.
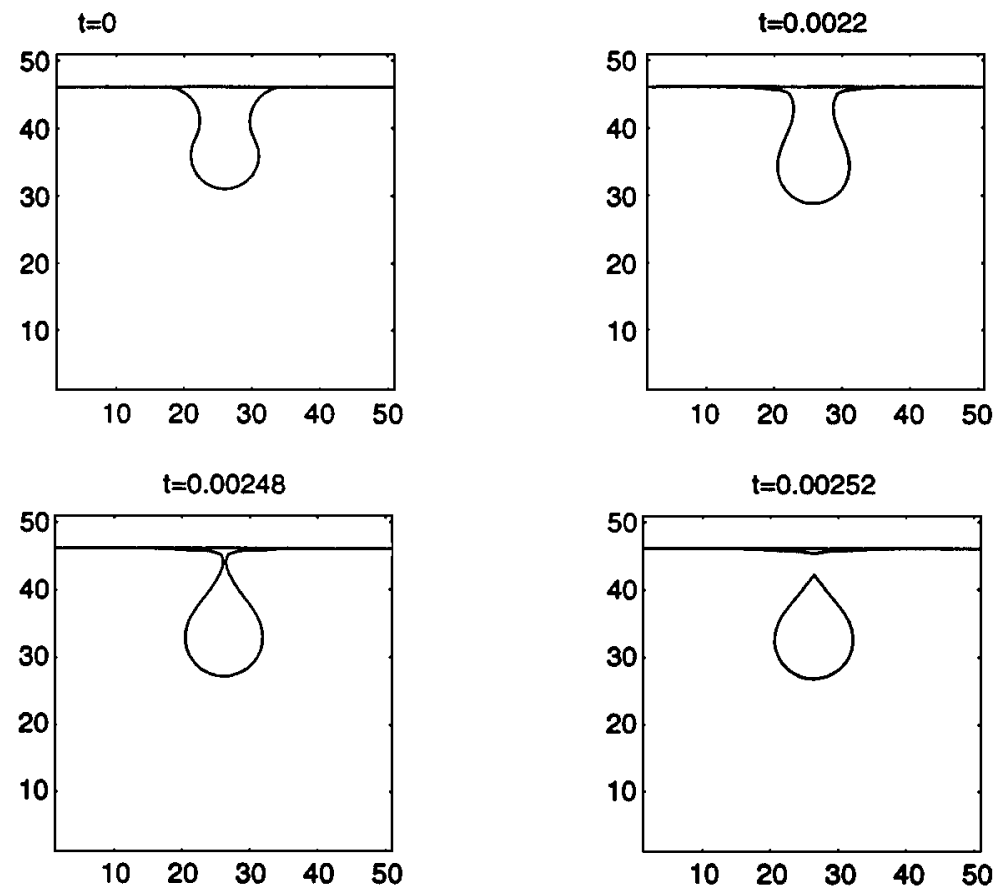

FIG. 11. Falling drop in 3D. Surface tension with unwetted wall $=0.5$, air $=1$, and gravity $=100$. 

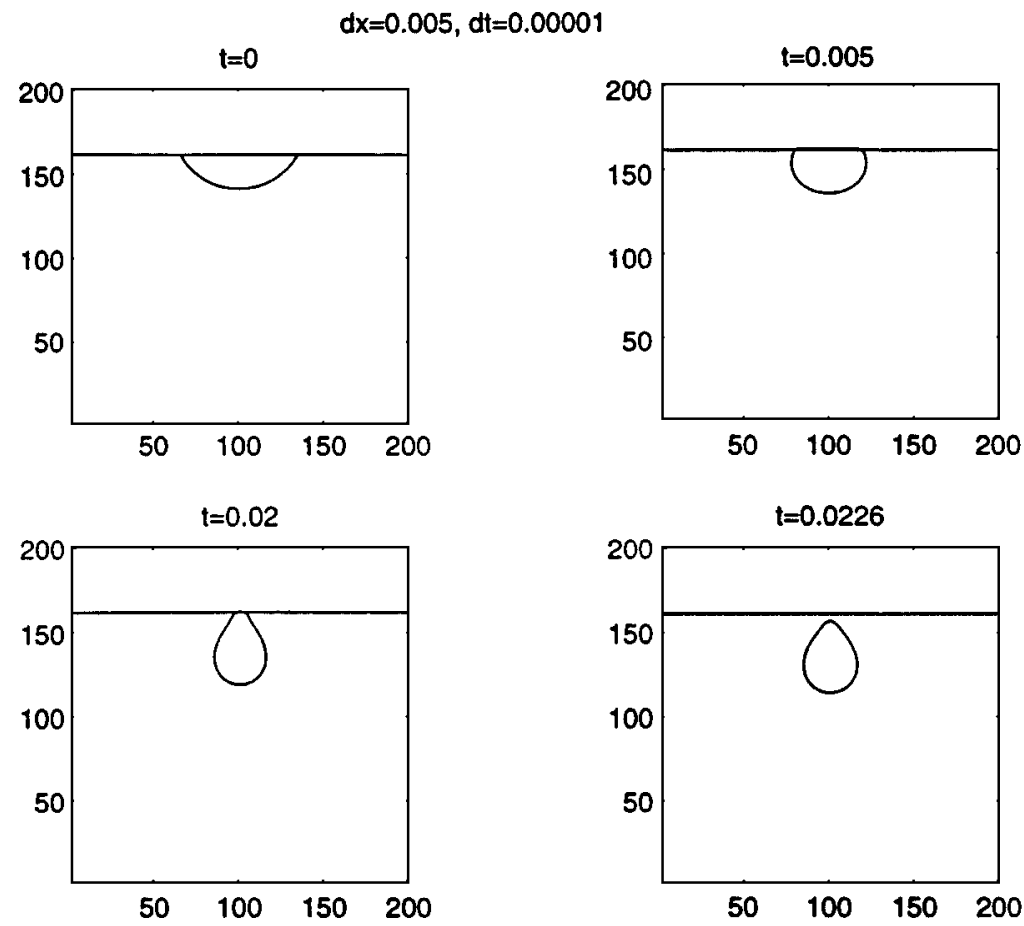

FIG. 12. Falling drop in 2D. Surface tension with wetted wall $=0.2$, air $=0.5$, and gravity $=100$.
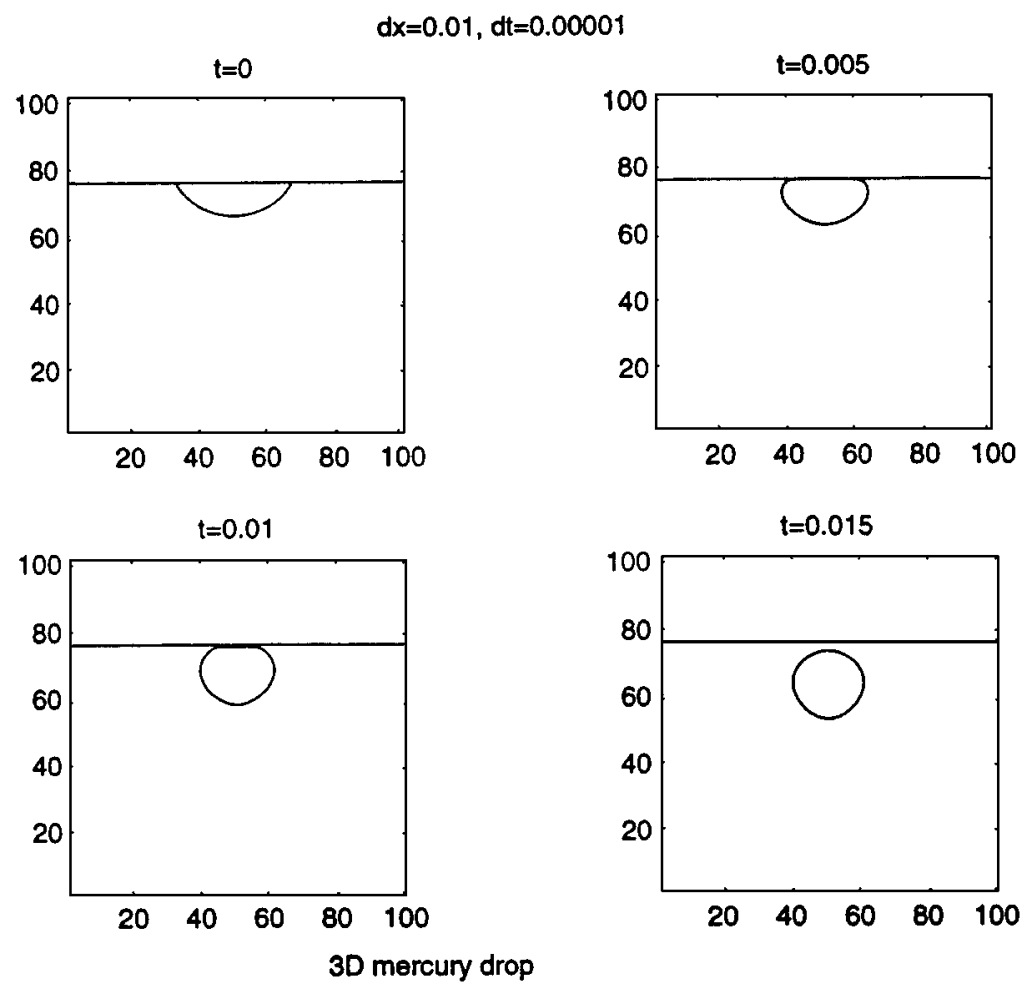

FIG. 13 . 

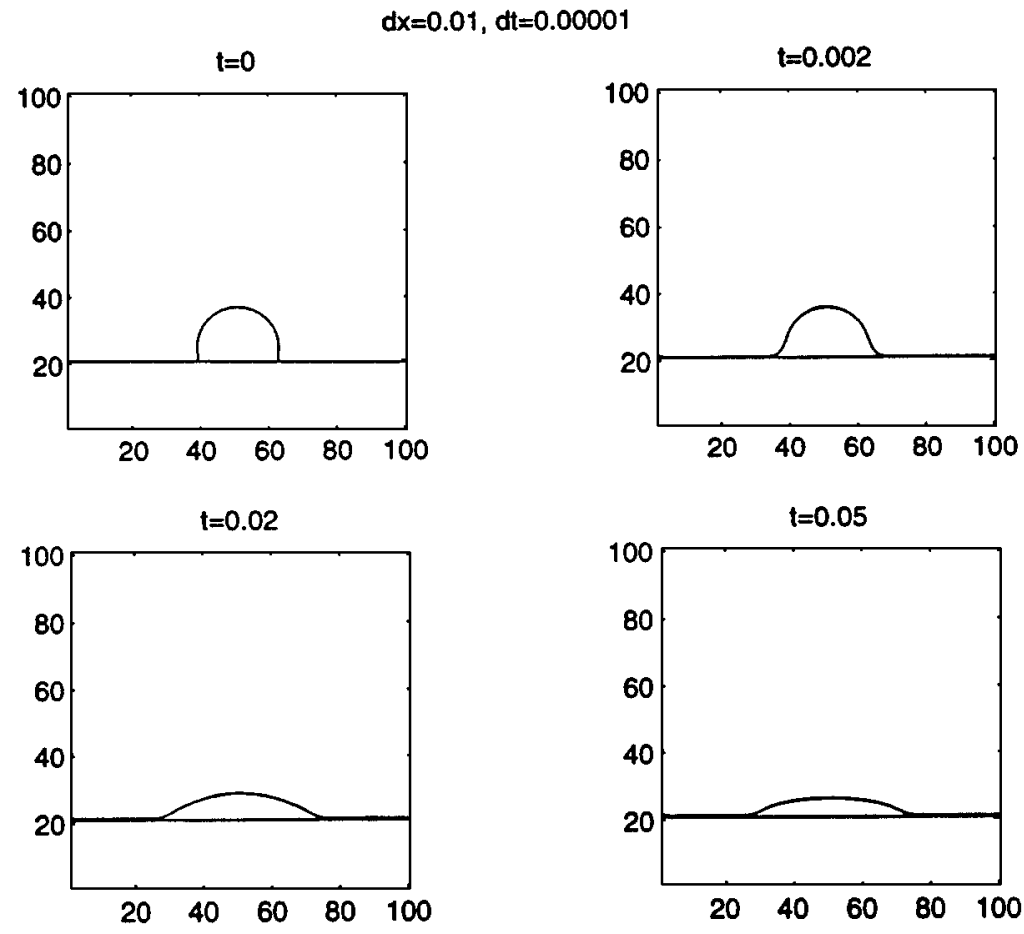

FIG. 14. Surface tension with unwetted wall $=0.2$, air $=0.3$, and gravity $=50$.
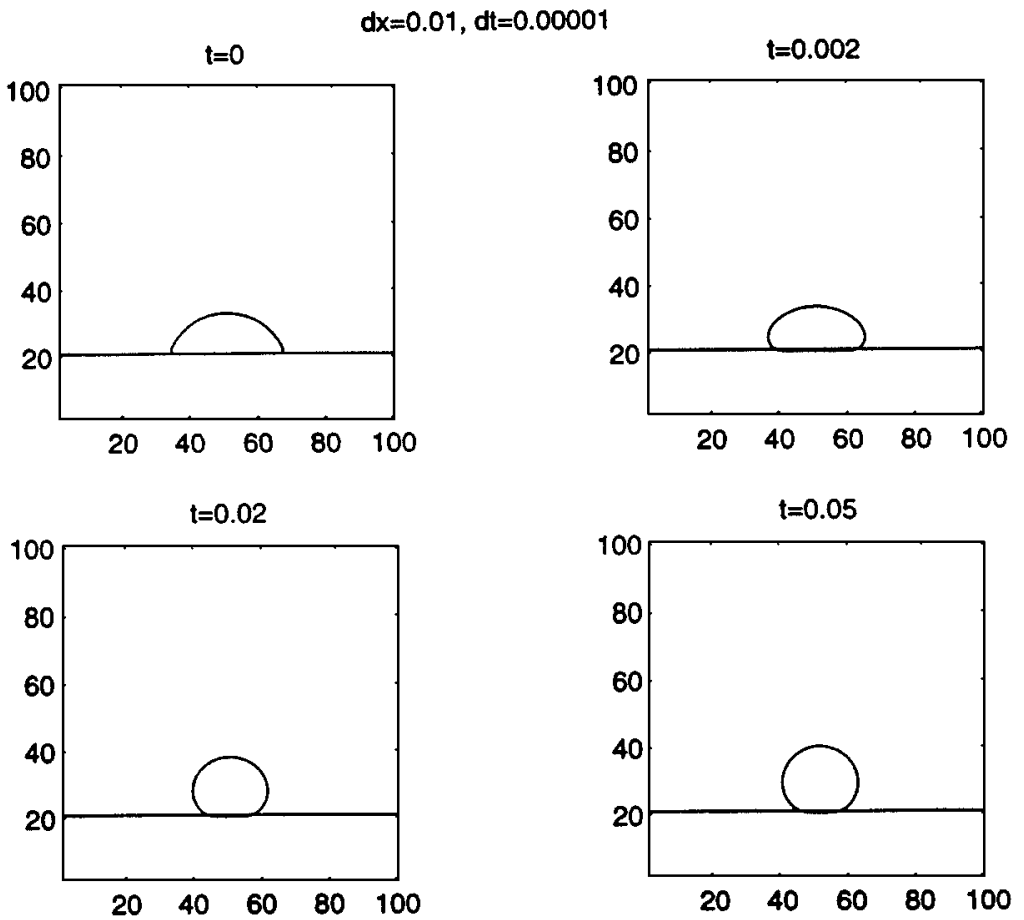

FIG. 15. Drop sitting on floor in $2 \mathrm{D}$. Surface tension with wetted wall $=0.5$, air $=0.6$, and gravity $=1$. 

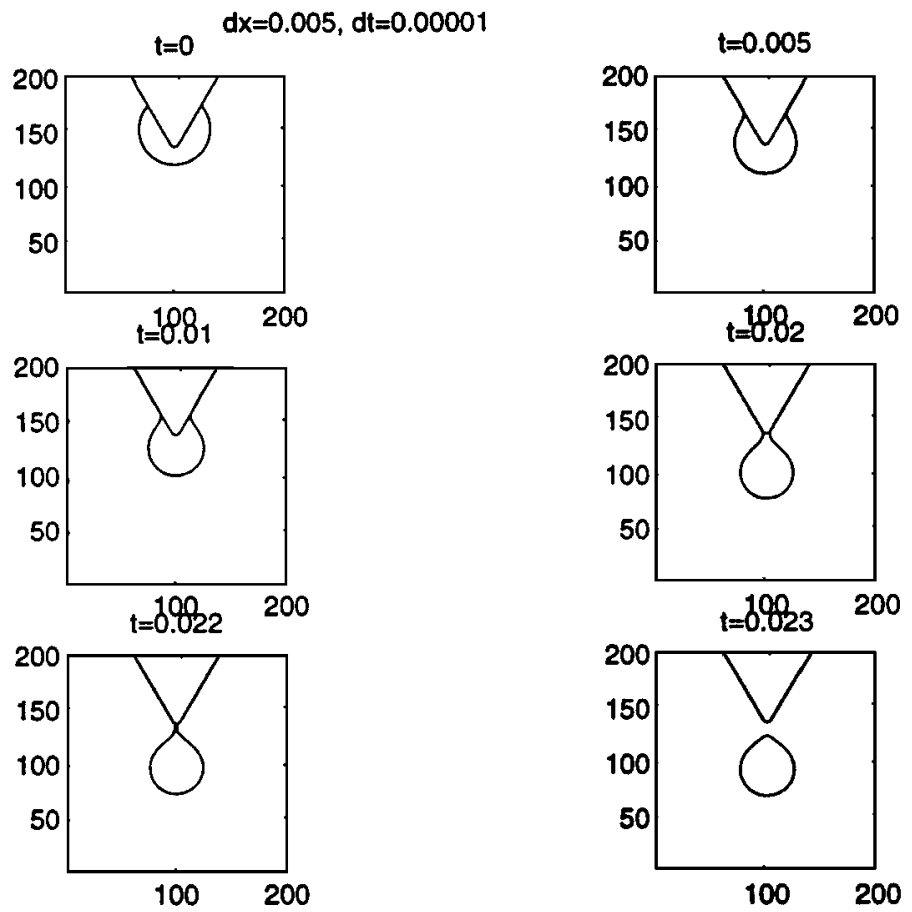

FIG. 16. Drop falling from wedge in $2 \mathrm{D}$. Surface tension with unwetted wall $=0.1$, air $=0.2$, and gravity $=100$.

balance is between the surface tension forces and gravity. Figures 16 and 17 show how the drops fall from the tip of a wedge in 2D. Figure 16 corresponds to the wetted case; Fig. 17 corresponds to the unwetted case.

Figures 18 and 19 show liquid flowing through a narrow funnel in 2D. Figure 18 shows the fluid going through the funnel. Due to the degeneracy of the boundary constraints, our numerical results show the liquid slightly penetrating the wall. By refining the grid in Fig. 19, we can see that the size of the penetration is also reduced. The size of the penetration is about one grid cell. In Fig. 20, we show a liquid at rest in a 2D funnel. Finally, in Fig. 21, we show a liquid going through an asymmetric funnel.

In Figs. 18 and 19 we print out the area at various times. We lose around $13 \%$ on the $200 \times 200$ grid calculation, $9 \%$ on the $300 \times 300$ grid calculation. However, we note that most of the loss (and occasional gain) occurs very early. For example, if we pick up the calculation at $t=0.02$ the loss is zero up to three decimal places in the refined calculation and $0.4 \%$ on the cruder grid. This is typical for our method.

\section{CONCLUSIONS}

We have developed a variational level set approach to capture the behavior of bubbles and droplet motions involving several phases. The method, as usual, handles topological changes easily and automatically and is relatively easy to program. The approach ignores inertial effects. These will be included in future work. The method is fast (overnight on a workstation for a three-dimensional problem) and local boundary conditions are treated via a penalty method. 

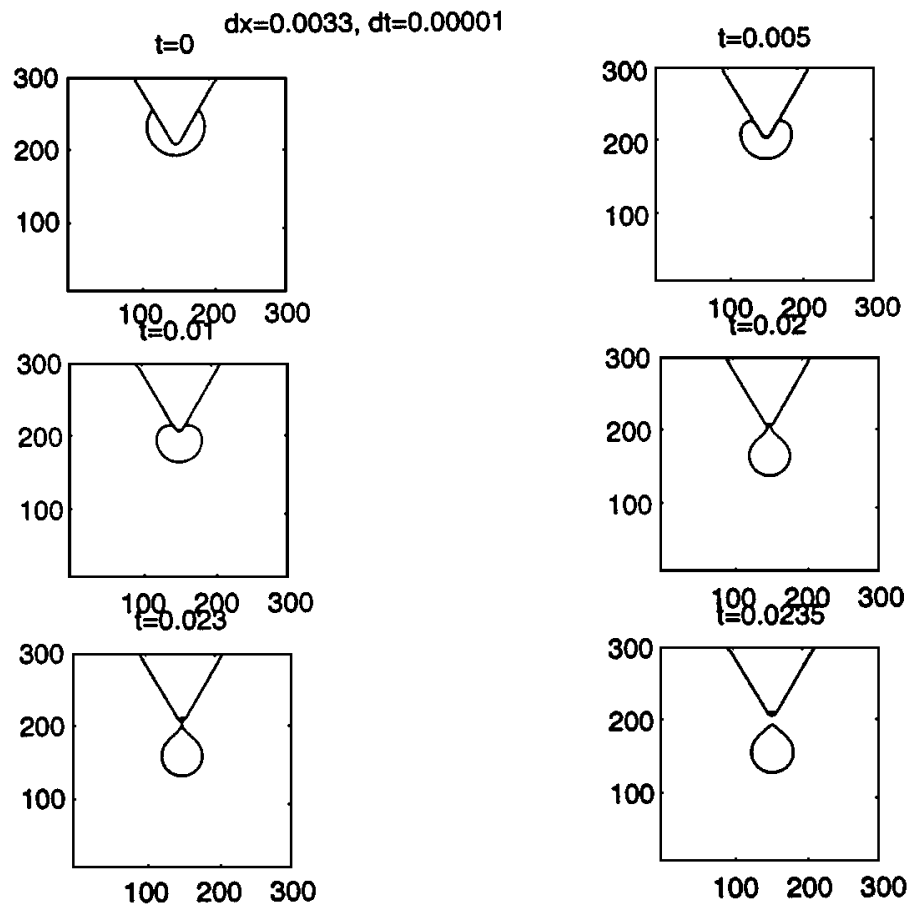

FIG. 17. Drop falling from wedge in $2 \mathrm{D}$. Surface tension with wetted wall $=0.1$, air $=0.2$, and gravity $=100$.

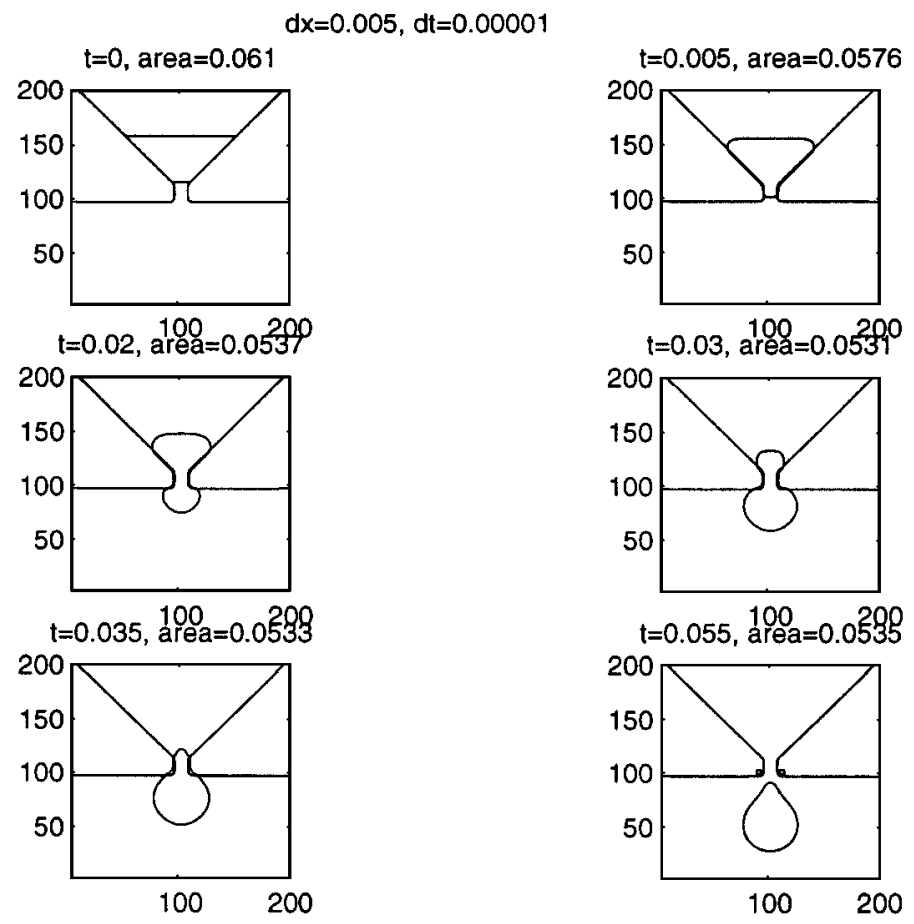

FIG. 18. Liquid flows through funnel in 2D. Surface tension with wall $=0.1$, air $=0.2$, and gravity $=100$. 

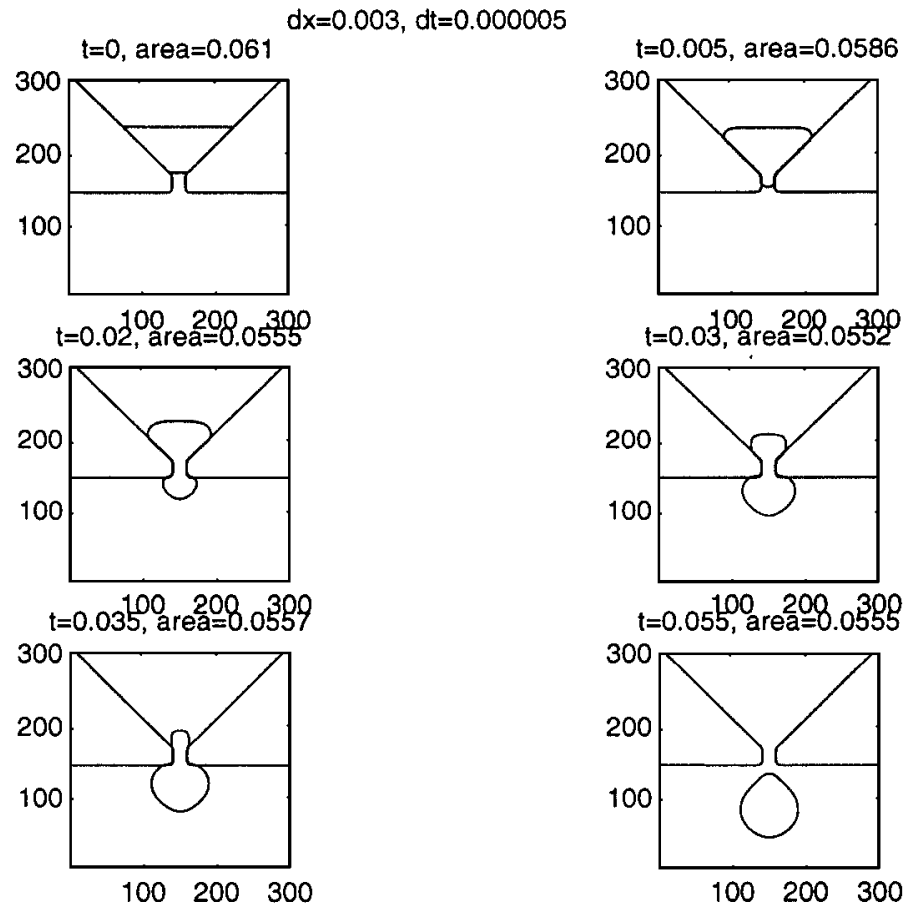

FIG. 19. Refined grid version of Fig. 18.
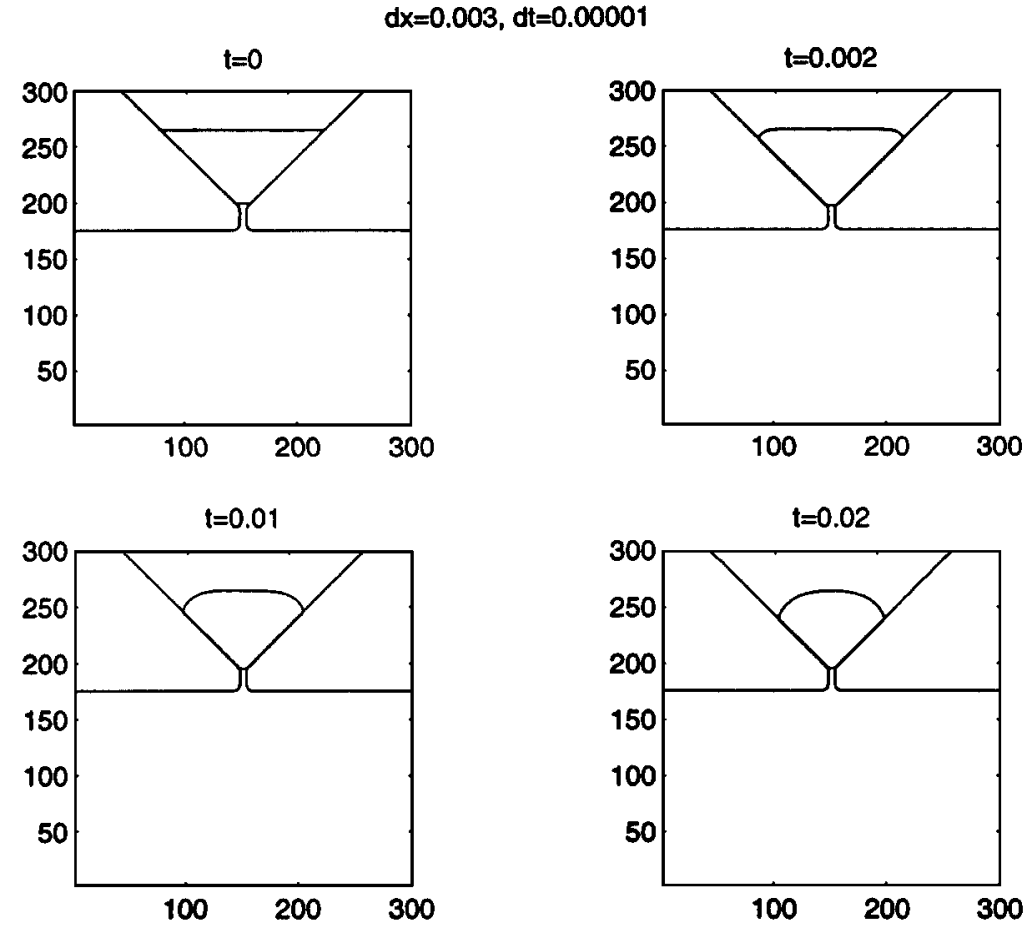

FIG. 20. Liquid at rest in funnel. 


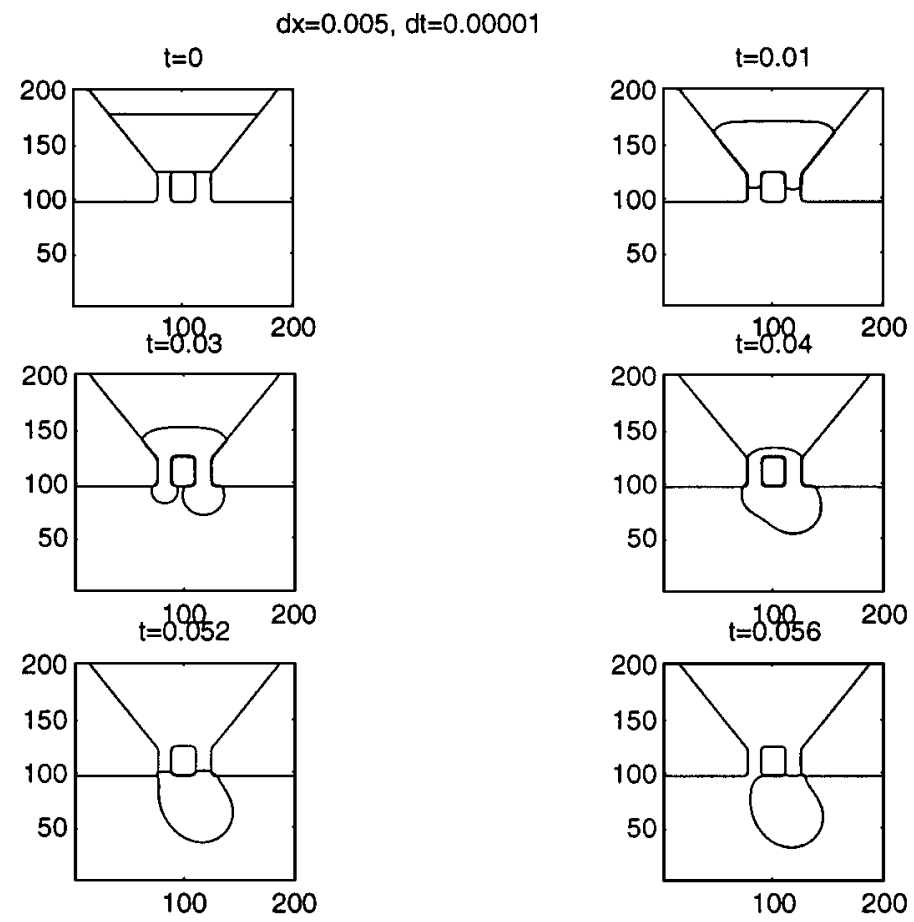

FIG. 21. Liquid flowing through asymmetric funnel. $g=50$, surface tension with the funnel $=0.1$, surface tension with the air $=0.2$.

Our results seem to reproduce the essential physics of the problems studied. Area loss for demanding problems is essentially nil, after the (dynamic) calculation settles down. Overall, we lose as much as $9 \%$ on a $300 \times 300$ grid calculation. We will address this issue, using a more sophisticated reinitialization scheme, in future work.

\section{REFERENCES}

1. D. Adalsteinsson and J. A. Sethian, A fast level set method for propagating interfaces, J. Comput. Phys. 118, 269 (1995)

2. Y. C. Chang, T. Y. Hou, B. Merriman, and S. Osher, A level set formulation for Eulerian interface capturing methods for incompressible fluid flows, J. Comput. Phys. 124, 449 (1996).

3. S. Chen, B. Merriman, S. Osher, and P. Smereka, A simple level set method for solving Stefan problems, J. Comput. Phys. 135 (1997), 8-29.

4. D. Chopp, Computing minimal surfaces via level set curvature flow, J. Comput. Phys. 106, 77 (1993).

5. E. Harabetian and S. Osher, Regularization of Ill-Posed Problems Via the Level Set Approach, UCLA CAM Report 95-41, 1995. [SIAM J. Appl. Math., to appear]

6. E. Harabetian, S. Osher, and C.-W. Shu, An Eulerian approach for vortex motion using a level set regularization procedure, J. Comput. Phys. 127, 15 (1996).

7. A Harten, B. Engquist, S. Osher, and S. R. Chakravarthy, Uniformly high order accurate essentially nonoscillatory schemes, III, J. Comput. Phys. 71, 231 (1987).

8. J. Hass, M. Hutchings, and R. Schlafly, The double bubble conjecture, Electron. Res. Announce AMS 1, 98 (1995).

9. M. Kang, A Level Set Approach for the Motion of Soap Bubbles with Curvature or Acceleration, Ph.D. thesis, UCLA, CAM Report 96-19, 1996. 
10. P. Mascarenhas, Diffusion Generated Motion by Mean Curvature, UCLA CAM Report 92-33, 1992.

11. B. Merriman, J. Bence, and S. Osher, Diffusion generated motion by mean curvature, in AMS Selected Lectures in Mathematics, The Computational Crystal Grower's Workshop, edited by J. Taylor (AMS, Providence, RI, (1993), p. 73. [UCLA CAM Report 92-18, 1992]

12. B. Merriman, J. Bence, and S. Osher, Motion of multiple junctions: A level set approach, J. Comput. Phys. 12, 334 (1994).

13. S. Osher, Subscale capturing in numerical analysis, in Proc. Int. Congress of Mathematicians, Zurich, 1994 (Birkhauser, Zurich, 1995), p. 1448.

14. S. Osher, Subscale capturing in CFD, in CFD Review (Wiley, Chichester, 1995), p. 182.

15. S. Osher and J. A. Sethian, Fronts propagating with curvature dependent speed: Algorithms based on a Hamilton-Jacobi formulation, J. Comput. Phys. 79, 12 (1988).

16. S. Osher and C.-W. Shu, High-order essentially nonoscillatory schemes for Hamilton-Jacobi equations, SIAM J. Numer. Anal. 28, 907 (1991).

17. G. Rosen, The gradient projection method for nonlinear programming. Part II. Nonlinear constraints, J. Soc. Indus. Appl. Math. 9, 514 (1961).

18. J. A. Sethian, Theory, Algorithms and Applications of Level Set Methods for Propagating Interfaces, CPAM, U.C. Berkeley Report, PAM-651, 1995. [ACTA Numerica, 1995]

19. X. D. Shi, M. P. Brenner, and S. R. Nagel, A cascade of structure in a drop falling from a faucet, preprint.

20. C.-W. Shu and S. Osher, Efficient implementation of essentially nonoscillatory shock-capturing schemes II, J. Comput. Phys. 83, 32 (1989).

21. M. Sussman, P. Smereka, and S. Osher, A level set approach for computing solutions to incompressible two-phase flow, J. Comput. Phys. 114, 146 (1994).

22. H.-K. Zhao, T. Chan, B. Merriman, and S. Osher, A variational level set approach to multiphase motion, $J$. Comput. Phys. 127, 179 (1996).

23. H.-K. Zhao, M. Kang, B. Merriman, D. Peng, and S. Osher, A PDE based fast local level set method, UCLA CAM Report 98-25, 1998.

24. H.-K. Zhao, J.-P. Shao, S. Osher, T. Chan, and B. Merriman, preprint. 